\title{
En el centenario de Allgemeine Psychopathologie
}

\author{
Xavier Urquiaga*
}

Ensayo

In memoriam Profesor doctor Dionisio Nieto Gómez

Hace exactamente 100 años, en el mes de julio del año 1913 y en vísperas del inicio de la Gran Guerra -acontecimiento que puede considerarse el principio histórico del siglo XX-, se publica en Alemania un libro extraordinario con el título de Psicopatología general. "Una guía para los estudiantes, médicos y psicólogos (Allgemeine Psychopathologie. Ein Leitfaden für Studierende, Ärzte und Psychologen)". Esta excepcional obra, junto con el Tratado de Psiquiatría de Emil Kraepelin, fundador de la nosología psiquiátrica moderna que la precedió, marca un hito en la historia de la psiquiatría, tal como ahora la conocemos y practicamos.

$\mathrm{Su}$ autor, Karl Jaspers, joven médico de apenas treinta años de edad, había nacido el 23 de febrero de 1883 en la ciudad de Oldenburgo, Alemania, en el seno de una familia liberal de clase media alta que por muchas generaciones había radicado en esa región. Una vez concluida la educación fundamental en su ciudad natal y ya desde entonces inclinado intelectualmente a la filosofía, para complacer a su padre inició en 1901 sus estudios de Leyes en la Universidad de Friburgo, mismos que a los pocos meses tuvo que interrumpir al presentar síntomas de incapacidad respiratoria, cuyo diagnóstico fue bronquiectasis. A pesar de las serias limitaciones ocasionadas por esta enfermedad, que continuarían a lo largo de su vida, después de ser sometido a tratamiento y un periodo de recuperación, su notoria tenacidad en todas las tareas que emprendió a lo largo de su vida determinó que en octubre de ese año reanudara sus actividades académicas. Fue así que se matriculó en la Universidad de Heidelberg para continuar sus estudios de Derecho; para cubrir las materias optativas que exigía la enseñanza de la época, diseñó un amplio y ambicioso programa que incluyó la asistencia a seminarios de filosofía, de arte y el de psicología experimental que entonces presidía Emil Kraepelin, Director de la Clínica Psiquiátrica de esa Universidad. Éste habría de ser su primer contacto con las ciencias de la salud mental.

Nuevamente, por su precaria salud se ve obligado a suspender sus estudios. En la primavera de 1902 viaja a Ita- lia y a su regreso a Alemania cursa el semestre de verano en Derecho, ahora en la Universidad de Munich. Sin embargo, ya que continuaba enfermo y su recuperación no era todavía aceptable, por consejo de sus médicos y como parte del tratamiento de su afección de vías respiratorias, en el mes de agosto se retira a las montañas de Sils Maria, en Suiza. En la tranquilidad del campo medita seriamente sobre su futuro y decide abandonar sus estudios en Derecho para dedicarse a la ciencia como un paso previo al estudio de la Filosofía que siempre fue la disciplina que más le interesó y a la que terminó por consagrar su vida entera. Para ello necesitaba convencer a su padre -quien financiaba sus estudios e insistía en que su hijo fuera abogado- de que su interés primario era la ciencia y no las leyes. Sus negociaciones tuvieron éxito y su padre aceptó continuar financiando sus estudios; en el semestre de invierno de ese mismo año se matricula como estudiante de Medicina en la Universidad de Berlín en donde cursa los dos primeros semestres de los doce que exigía esa carrera. Su enfermedad y la circunstancia de la relativa lejanía entre la Universidad y el sitio de su alojamiento limitaban su desempeño como estudiante, por lo que decide mudarse a la Universidad de Gotinga en donde continuará sus estudios de Medicina y permanecerá los tres años siguientes. Pero por consideraciones relacionadas de nuevo con su salud y también por sus intereses científicos e intelectuales, en el verano de 1906 Jaspers retorna a la Universidad de Heidelberg en donde concluye sus estudios de Medicina y en enero de 1908 presenta el examen de Estado y se gradúa "suma cum laude".

Con el propósito de dedicarse a la Psiquiatría, continúa su adiestramiento médico como pasante de Medicina en la Clínica Psiquiátrica de la propia Universidad de Heidelberg, hospital cuya dirección había dejado Emil Kraepelin años atrás -al ser nombrado Profesor de Psiquiatría en la Universidad de Munich- y que en 1908 estaba bajo la dirección del prestigiado neuroanatomista Franz Nissl. Sin embargo, pronto considera que si bien tenía la oportunidad de pro-

* Colegio de Psiquiatras del Estado de Yucatán. Mérida, Yucatán, México.

Miembro del Comité Editorial de SALUD MENTAL. 
fundizar en las historias clínicas de los pacientes, al capítulo de la terapéutica no se le daba casi ninguna importancia. "La gran equivocación de esta clínica es que no se aprende terapia. Sobre todo, una suerte de nihilismo terapéutico se estima aquí. Sin embargo, no es tan mala en el sentido de que si eres bueno en diagnóstico la terapia representa la parte más fácil. En cuanto al diagnóstico no podría tener instrucción mejor que aquí tanto por la cantidad de material como por el abordaje científico muy crítico que prevalece en la Clínica". 1

Adicionalmente, según recuerda en sus notas autobiográfícas, ${ }^{2}$ le pareció que como era común a otros hospitales alemanes, no sólo la enseñanza del tratamiento era nula, sino que tampoco se hacía investigación científica. No obstante esto, interesado como estaba en los trastornos mentales, trató de aprender todo lo que se le presentaba. Pronto percibió que había una confusión enorme en cuanto a la terminología empleada por los psiquiatras provenientes de diferentes escuelas, factor que hacía muy difícil el entendimiento entre ellos. "Me pareció que un origen de este revoltijo intelectual descansaba en la naturaleza del caso. El objeto de la psiquiatría era el hombre, no sólo su cuerpo... Nuestro tema era también el de Geisteswissenschaften (las ciencias humanas). Tenían que desarrollar los mismos conceptos, sólo que con mucho más sutileza y definición. Un día escuchábamos un discurso en estado confusional o un hablar paranoico y entonces le dije a Nissl: 'Tenemos que aprender de los filólogos '. Fue cuando empecé a buscar qué nos podían ofrecer la Filosofía y la Psicología... Ésta era la situación en 1911 cuando Wilmanns y el editor Ferdinand Springer me pidieron escribir la Psicopatología General."2

Independientemente de estos obstáculos para su preparación en Psiquiatría, Jaspers enfrentó otro problema que probablemente determinó su fugaz paso por ella, que pudo haberse prolongado por mucho más tiempo de no haberlo padecido. Su incapacidad respiratoria le impedía hacerse cargo de las exigencias laborales en la Clínica y limitaba en mucho la necesidad imperiosa de estar en contacto con los enfermos, imposibilitándole así la obtención directa de información detallada que siempre consideró de capital importancia, tanto para la investigación como para la práctica psiquiátrica. No obstante lo anterior, esto le permitió llevar a cabo los trabajos necesarios para escribir y defender su tesis "Nostalgia y delito"3 en diciembre de 1908, con la que obtuvo el grado de Doctor y que fue publicada al año siguiente.

De nuevo, por su incapacidad física se vio obligado a interrumpir su trabajo con los enfermos asistidos en la clínica y a principios de 1909, ya como residente en psiquiatría (asistente voluntario sin sueldo) y sin haber dejado sus intenciones de ser psiquiatra, convence a Franz Nissl de que bajo su supervisión se le permita continuar trabajando en investigación en la biblioteca de la Clínica, haciendo sólo algunas suplencias cuando alguno de sus colegas no asistía o no estaba disponible. Franz Nissl aceptó.
Esta nueva ocupación permitió a Jaspers consagrase por entero a escribir varios trabajos de singular importancia en psicopatología y que fueron publicados en la revista Zeitschrift für die gesamte Neurologie und Psychiatrie, fundada por Alois Alzheimer en 1910. Ellos son, en orden cronológico: Delirio celotípico, contribución al problema: ¿Desarrollo de una personalidad o Proceso?(1910), ${ }^{4}$ conceptos fundamentales en su obra de psicopatología; Los métodos de medición de la inteligencia y el concepto de demencia (1910), ${ }^{5}$ del que estuvo particularmente orgulloso; Análisis de las percepciones equívocas (vivacidad y juicio de realidad) (1911); ${ }^{6}$ La corriente de investigación fenomenológica en psicopatología (1912) ${ }^{7}$ y Relaciones causales y "comprensibles" entre destino y psicosis en la demencia precoz (esquizofrenia) (1913). ${ }^{8}$

La culminación de estos trabajos en este periodo de la vida de Karl Jaspers fue su monumental obra Psicopatología General que principió a escribir en 1911 a solicitud del editor Ferdinand Springer y de su colega Karl Wilmanns, quien en 1918 sucedió a Franz Nissl en la dirección de la Clínica Psiquiátrica de Heidelberg hasta 1933, año en el que fue el primer profesor universitario de gran prestigio en ser expulsado de su puesto en una Universidad alemana por razones políticas, con lo que terminó uno de los periodos más luminosos de dicho Hospital.

Una vez publicada la Psicopatología General, la presenta como tesis con lo que consigue ser habilitado para la docencia y obtiene el nombramiento de conferencista sin sueldo en Psicología en la Facultad de Filosofía de Heidelberg. Tres años después, en 1916, es ascendido a profesor asistente en Psicología en la misma Facultad. En 1919 publica Psicología de las concepciones del mundo, tratado en el que propone los temas filosóficos fundamentales que desarrolló a lo largo de su vida y que ahora es considerado el primer libro de filosofía existencialista que se publicó, puesto que precedió a El ser y el tiempo (1927) de Martin Heidegger. A partir de entonces, la trayectoria intelectual y académica de Jaspers se separa de la Medicina y de la Psiquiatría y se dirige a la Filosofía, disciplina de la que se ocuparía a lo largo de toda su vida y de la que ya nunca más se apartó.

En 1921 obtuvo la cátedra de Filosofía en la Universidad de Heidelberg y en 1932 publicó el libro Filosofía, que se considera su obra filosófica más importante. Permaneció en este puesto hasta 1937, año en el que el régimen de Hitler lo expulsó de la Universidad ya que, fiel a su propia filosofía, era un importante, prestigiado, firme y radical opositor al nacional socialismo. A ello se añadía el hecho de estar casado con Gertrud Mayer, judía alemana con la que había contraído matrimonio en 1910. Durante la dictadura hitleriana que lo condenó al ostracismo y prohibió todas sus publicaciones, permaneció retirado en la ciudad de Heidelberg, pero continuó su actividad intelectual. Esto le permitió, después de muchos años, volver a la Psiquiatría al hacer una revisión profunda de su Psicopatología General, que pudo publicar hasta 1946 por la prohibición que había pesado sobre 
sus obras. Con muy pocos cambios y notas en posteriores ediciones es la versión que conocemos, traducida al español y publicada por primera vez en Argentina en $1970^{\circ}$ y luego, en México, en versión revisada, en 1993. ${ }^{10}$

Sus intentos para salir de Alemania a Inglaterra y a Suiza le fueron condicionados a la permanencia de su esposa en Alemania, exigencia que de ninguna manera aceptó y qué, incluso, lo llevó a hacer un pacto suicida con ella en caso de que fuera deportada a un campo de concentración. Esto no llegó a suceder porque, a pesar de ya estar ella incluida en la lista del siguiente convoy que partiría de Heidelberg a algún campo de exterminio, la ciudad fue liberada al tomarla el ejército norteamericano el $1^{\circ}$ de abril de 1945.

El 15 de abril de ese año es reinstalado en su cátedra de Filosofía y pronuncia el Discurso Inaugural de reapertura de la Facultad de Medicina. A partir de entonces publica un gran número de libros y ensayos filosóficos que lo hicieron acreedor a muchos premios y distinciones académicas. En 1948 es nombrado Profesor de Filosofía en la Universidad de Basilea en donde permanece hasta su muerte acaecida el 26 de febrero de 1969. Actualmente se considera a Karl Jaspers y a Martin Heidegger los máximos exponentes de la filosofía existencialista de Alemania.

La Psicopatología General de Jaspers no es un tratado de Psiquiatría; tampoco es un libro de Filosofía. Es un libro de Psicopatología, novedoso en su concepción, en su contenido y en su estructura, que le confiere características especiales y que lo sitúa entre la ciencia médica y la Filosofía. Para Jaspers, tener experiencia científica era una exigencia necesaria para hacer filosofía y constituyó un paso previo para transitar a ella. Esta manera de su proceder intelectual marca la evolución de su pensamiento que lo llevó finalmente a lo que fue siempre su interés primario: la Filosofía.

La Psicopatología General no fue el primer libro que se escribió con ese título. Ciertamente, como el mismo Jaspers lo señala en la Introducción de su obra, en Alemania dos libros de psicopatología precedieron al suyo, el de Herman Emminhghaus, publicado en $1878^{11}$ y el de Störring, que apareció en $1900 .{ }^{12}$ Del primero, Jaspers dice: "Su método es puramente descriptivo y muestra las actitudes generales no probadas de la Medicina basadas en las ciencias naturales de su tiempo"13 y sobre el segundo, advierte que es fundamentalmente teórico: "pero ante la enorme variedad de la realidad psíquica, el libro de Störring ofrece soluciones muy limitadas". ${ }^{14}$

Sin embargo, y sin subestimar el mérito de esas obras, Jaspers consideró que no cumplían con lo que a su juicio era el principal propósito de la Psicopatología puesto que para él: "Una psicopatología general no es sólo la exposición didáctica de lo ya existente, más bien realiza un trabajo consciente en la ordenación del todo. Todo psiquiatra se caracteriza por la especie del ordenamiento en que tiene una imagen total más o menos compleja, móvil o rígida. Un libro sobre Psicopatología quiere cooperar en ese cuadro total o en el modo de pensar del todo, en donde todos los métodos particulares tienen su sentido y sus límites. Libros que aspiran justamente a una exposición total, tienen por tanto su importancia decisiva por el modo como ven el todo y como hacen aparecer ese todo en la sistemática visible y en la dirección del pensamiento". ${ }^{15}$

Así pues, para Jaspers el objeto de la Psicopatología es algo muy distinto, más amplio, variado y profundo que el de sus predecesores: "Es el acontecer psíquico realmente consciente. Aunque su principal preocupación son los hechos patológicos, es también necesario conocer lo que en general experimentan los seres humanos y cómo lo experimentan; en resumen se ocupa de la totalidad de la realidad psíquica. Es necesario no sólo examinar las vivencias sino también las causas y las condiciones en que aparecen, así como las relaciones y los modos en los que la experiencia se manifiesta". ${ }^{16}$

Mucho se ha dicho que la Psicopatología General es un libro de difícil lectura y que, en gran medida, a esto se debe -excluyendo la Psiquiatría alemana sobre la que tuvo gran influencia desde su publicación- su tardía aceptación e influjo sobre las teorías psiquiátricas y sobre la práctica misma de la Psiquiatría en otros países porque, en palabras de Michael Shepherd: "su argumentación es densa y expuesta en forma difusa, bastante difícil en el original alemán y con frecuencia también comprensiblemente difícil en otro idioma a pesar de los heroicos esfuerzos de sus traductores... Quizá la dificultad principal que presenta este libro, es que no encaja en un modelo familiar reconocible para el lector ${ }^{\prime \prime}{ }^{17}$

Ciertamente, para leer este libro y hacer más provechosa su lectura, es importante tener presente su peculiar estructura. En este sentido, como bien señala S. Nassir Ghaemi, ${ }^{18}$ otra vez es el eminente psiquiatra inglés Michael Shepherd, dedicado estudioso de Jaspers, "quien probablemente mejor ha captado su naturaleza" cuando afirma que: "debe considerarse como un mapa intelectual, una guía para una serie de temas separados, pero relacionados, del conocimiento y que se identifican en el índice... El propósito primario del libro no es, sin embargo, presentar un estudio ordenado sino una visión general diseñada, en palabras de Jaspers, 'para desarrollar y ordenar el conocimiento guiados por los métodos por medio de los cuales se gana para aprender a conocer el proceso del conocimiento y por lo tanto aclara el material'. La consecución de este objetivo depende primariamente de la clarificación de una serie de conceptos que tradicionalmente son ignorados o excesivamente simplificados en la literatura psiquiátrica. Para tratar debidamente temas tales como la relación mente-cuerpo, el papel de la investigación científica, los principios de la clasificación, la personalidad, la dicotomía subjetivo-objetivo o las nociones de salud y enfermedad se exige un conocimiento de la historia de las ideas en otras disciplinas. Es aquí donde Jaspers introduce sus propias ideas recurriendo a una voluminosa tradición de las teorías filosóficas y sociales para apoyar estos problemas perennes en relación con la psicopatología."19 
Resumir la Psicopatología General de Jaspers es un intento vano; ni siquiera una síntesis del libro puede dar una idea precisa de lo que aporta. Intentarlo tampoco tiene mucho sentido pues por excelente que resultara la síntesis, necesariamente se perderían muchas de las ideas profundas y sutiles que en él se expresan y se caería con facilidad en la superficialidad.

El libro se divide en seis partes que son precedidas por la Introducción y concluye con un Apéndice. Sin embargo, para tener una cabal idea de su contenido quizá el mejor camino sea recurrir al propio Jaspers y dejar que él mismo lo exprese. En la Introducción lo resume así:

"En la primera parte aparecen los hechos típicos particulares empíricos de la vida psíquica. Las vivencias subjetivas y los estados somáticos, los rendimientos objetivos y los hechos significativos se manifiestan sucesivamente en expresión mundo y obra (por ejemplo expresiones, producciones y las propias palabras de los enfermos). Toda esta parte ejercita al mismo tiempo los órganos de aprehensión del psicopatólogo y muestran los resultados inmediatos".

"En la segunda y en la tercera partes nos dedicamos a las relaciones de la vida psíquica y, más precisamente en la segunda, a las comprensibles y en la tercera a las causales. Las relaciones no son conocidas directamente por la admisión de los hechos, sino mediatamente, en la investigación, por la verificación de los hechos. Estas dos partes ejercitan también los órganos de investigación del psicopatólogo. Ya que el hombre entre espíritu y naturaleza es al mismo tiempo ambas cosas, para su conocimiento son exigibles igualmente todas las ciencias. Lo que se investiga en la segunda parte, presupone un dominio de las ciencias del espíritu, lo que se indaga en la tercera, un dominio de la biología".

"En la cuarta parte, después de lo predominantemente analítico, sigue algo predominantemente sintético. Se trata de saber cómo es concebible la totalidad de la vida psíquica. Lo que aquí aparece a la vista, despierta la concepción total del clínico. Éste ve al hombre entero individual, piensa en la unidad nosológica, en su diagnóstico, en la constitución que lo entraña todo, y en la biografía, en cuya totalidad tan sólo se muestra cada individuo".

"La quinta parte considera a la vida psíquica anormal sociológicamente y en la historia. La psiquiatría es distinguida del resto de la medicina también por el hecho de que el alma humana recibe su sello entero de la circunstancia de que el hombre no es sólo una criatura natural, sino un ser cultural. Los procesos psíquicos morbosos dependen en su contenido y en su forma, del círculo cultural y repercuten en él. La quinta parte presenta la visión histórica de la realidad humana".

"En la sexta parte llegamos a una discusión terminal sobre el todo del ser humano. En esta parte no son hechas comprobaciones empíricas ya, sino que más bien tiene lugar una reflexión filosófica. Las totalidades específicas que tuvieron en cada capítulo un sentido directivo, son todas relativas.
Tampoco la concepción general del clínico abarca empíricamente el todo del ser humano. Siempre es el hombre más aún de lo que se reconoce en él. La discusión final no aumenta por tanto nuestro saber, sino que esclarece nuestra actitud filosófica básica en la que realizamos todo saber y todo conocer del hombre".

"El tema de este libro consiste en mostrar lo que sabemos. Sólo en el apéndice son caracterizadas fundamentalmente las tareas prácticas. Se traza una breve reseña de la psicopatología como ciencia" ${ }^{20}$

Como se puede ver en esta reseña del propio Jaspers, su Psicopatología General trata de temas fundamentales para la Psiquiatría, cuyo cuidadoso y sereno análisis es contrario a todo dogmatismo, a lo que Jaspers de manera radical se opuso siempre. En ella se plantean los fundamentos de la Psicopatología considerando al ser humano en su totalidad, como exige cualquier análisis que se haga de él y que pretenda un auténtico conocimiento del hombre sano o enfermo.

Es importante dejar claro que en su Psicopatología General Jaspers utilizó únicamente parte del método fenomenológico puesto que "aceptó sólo el primer paso de la fenomenología, el momento descriptivo y se negó a dar el paso siguiente, el de la búsqueda de las esencias, por considerarlo de carácter filosófico o metafísico y por ende no científico". ${ }^{21}$

Por tal motivo, advierte que: "Hay un malentendido cuando se ha designado mi libro como 'obra principal de tendencia fenomenológica'. La actitud fenomenológica es un punto de vista y se ha expuesto detalladamente en un capítulo de este libro. Pero la idea del libro es justamente que sólo es un punto de vista e incluso un punto de vista subordinado". ${ }^{22}$ Así pues, Jaspers sólo aplicó a la psiquiatría un método tomado de la Filosofía pero eso no significa que haya hecho filosofía de la psiquiatría, como algunos pretenden. Aplicar un método filosófico a una ciencia no convierte a nadie en filósofo pues aceptar esto equivaldría a sostener que hace filosofía quien aplica el método de la lógica de Aristóteles a su cotidiano pensar.

Más adelante en el libro puntualiza Jaspers la utilidad que tiene para la Psicopatología el método fenomenológico cuando dice: "La fenomenología tiene varios propósitos: proporciona una descripción concreta de los estados psíquicos que los enfermos realmente experimentan y muestran para ser observados; revisa la interrelación entre ellos, los delimita con toda la precisión posible, los diferencia y les proporciona una terminología adecuada. Ya que nunca podemos percibir las experiencias psíquicas de otros de una manera directa, como ocurre con los fenómenos físicos, sólo podremos tener alguna representación de ellos. Tiene que haber un acto de empatía, de comprensión, al que se puede agregar, según lo exija el caso, una enumeración de las características externas del estado psíquico o de las condiciones bajo las cuales ocurre, podremos hacer comparaciones definidas o recurrir al uso de símbolos o a algún tipo de manejo sugestivo de los datos. Nuestra principal ayuda en todo esto proviene de las propias 
autodescripciones de los enfermos, que pueden ser evocadas y probadas en el curso de las conversaciones personales; de todo esto obtenemos datos con mejor definición y claridad. Las descripciones escritas por los pacientes pueden tener un contenido abundante pero no podemos hacer otra cosa más que aceptarlas. Una experiencia es mejor descrita por la persona que la ha sufrido. Las observaciones psiquiátricas que se aparten con sus propias elaboraciones sobre lo que el enfermo está sufriendo nunca las sustituirá".23

"Lo que se necesita es una tranquila inmersión en los hechos de la vida psíquica sin adoptar ninguna actitud específica hacia ellos. Los seres humanos tienen que ser observados de manera imparcial, con vivo interés y sin hacer ningún tipo de valoración". ${ }^{24}$

Sin embargo, advierte Jaspers, "La fenomenología \{sólo\} nos da una serie de fragmentos de lo psíquico realmente vivenciado... \{pero como\} los actos y mundos de los enfermos y sus manifestaciones mentales muestran siempre otro tipo de hechos, preguntamos en qué relación están todos ellos". ${ }^{25}$

Ante este cuestionamiento, cuya respuesta es fundamental para que la Psicopatología no se limite a la simple identificación y recolección de hechos psíquicos captados fenomenológicamente, Jaspers, con sorprendente claridad y sencillez resuelve este grave problema al aplicar a los fenómenos psíquicos el método de la comprensión y la explicación propuesto por Wilhelm Dilthey en $1894 .{ }^{26}$ En efecto, Dilthey había sugerido años atrás que este método era el adecuado para el estudio de las ciencias del espíritu (ciencias humanas), que eran susceptibles de comprensión y entre las que incluía la psicología y las ciencias naturales, accesible su estudio por medio de la explicación y que entre otras comprendían la biología. De su obra se acuñó el conocido aforismo: "La mente se comprende, la naturaleza se explica".

Ciertamente, la vida psíquica de toda persona es una sucesión de hechos, vivencias y acontecimientos que confluyen en el tiempo y las circunstancias particulares que a cada quien le toca vivir. Estas condiciones determinan en gran medida sus características particulares, únicas e irrepetibles que las distinguen de las demás y que conforman el sentido de su propia historia. Pero es evidente que, al no tratarse de hechos aislados e inconexos sino que por el contrario se interrelacionan entre sí de múltiples formas y maneras, la vida psíquica de la persona se organiza y manifiesta plena de sentido, es decir se comprende. Esta realidad que sin el menor esfuerzo verificamos todos los días en nosotros mismos y también en los demás, integra la propia, personal e irrepetible biografía que por sus características únicas le confiere la identidad, singularidad e individualidad que distingue a todo ser humano.

En rigor, el propio Jaspers ya había ensayado con fructíferos resultados el método de la comprensión y la explicación de los fenómenos psíquicos en otros trabajos ${ }^{4,7,8}$ que escribió en la misa época que su Psicopatología General. Sin embargo, es aquí en donde resume sus ideas y las propone como un método para definir con mucho más precisión lo que les ocurre a los enfermos mentales:

"Para eludir ambigüedades, empleamos la expresión comprender (verstehen) siempre para la visión de lo psíquico desde dentro. Al hecho de conocer relaciones causales objetivas, que sólo es visto desde fuera, no lo llamamos nunca comprender, sino siempre explicar (erklären)" ${ }^{27}$

"Mientras en las ciencias naturales sólo pueden ser halladas relaciones causales, en psicología, nuestra inclinación por el conocimiento es satisfecha en la captación de una especie muy distinta de relaciones. Lo psíquico ‘surge' de lo psíquico de una manera comprensible para nosotros. Quien es atacado se enoja y realiza actos de defensa, el que es engañado se vuelve desconfiado. La manera en que tal surgimiento se lleva a cabo es comprendida por nosotros, nuestra comprensión es genética. Así comprendemos reacciones vivenciales, el desarrollo de pasiones, la aparición del desvarío, comprendemos el contenido del sueño y del delirio, de los efectos de la sugestión, comprendemos una personalidad anormal en su propia relación esencial, comprendemos el curso fatal de una vida, comprendemos cómo el enfermo se comprende a sí mismo, y cómo la manera de esa comprensión de sí mismo se vuelve un factor del desarrollo psíquico ulterior" ${ }^{28}$

Más adelante, siguiendo esta misma línea de pensamiento, Jaspers proporciona un panorama completo y preciso del método por él empleado, al distinguir dos formas del comprender genético, a saber: la "comprensión racional", que es puramente fenomenológica y descriptiva: "Por ejemplo, cuando los pensamientos pueden ser entendibles porque emergen unos de otros de acuerdo a las reglas de la lógica y las conexiones se entienden racionalmente (comprendemos lo hablado)"29 y la "comprensión empática" (einfühlden), que es aquella que surge exclusivamente de los fenómenos psíquicos, tiene su origen en ellos mismos y permite relacionarlos entre sí: "Pero cuando comprendemos los contenidos mentales como surgidos de los estados de ánimo, deseos y temores del que piensa, comprendemos primero de modo psicológico o empático (comprender del que habla)". 30 "Si el comprender racional conduce siempre a la comprobación de que el contenido psíquico es simplemente una conexión racional, comprensible sin ayuda de la psicología, el comprender empático, por otra parte, siempre nos conduce directamente a las conexiones psíquicas mismas. Si la comprensión racional es sólo un medio auxiliar de la psicología, la comprensión empática lleva a la psicología misma". ${ }^{31}$

Lógica consecuencia de lo anterior es que en las ciencias naturales la comprensión del hecho observado no es operante ya que si ellas no son accesibles a la comprensión sólo se podrán explicar. Añade, por otra parte, que si bien la explicación de los fenómenos naturales no tiene límite, "la comprensión en cambio encuentra fronteras en todas partes"... 
"La existencia de predisposiciones psíquicas, especiales, las reglas de la adquisición y pérdida de las disposiciones de la memoria, la consecuencia del estado psíquico total en las diferentes partes de la vida y todo lo demás, que podemos resumir como subestructura de lo psíquico, es frontera para nuestra comprensión". ${ }^{32}$ En el mundo físico todo fenómeno observado necesariamente tiene una explicación y el hecho de que en determinadas circunstancias ésta no pueda darse, de ninguna manera significa que no exista, sino simplemente que de momento no se conoce pero que con toda probabilidad en el futuro se encontrará.

Para Jaspers el problema psicopatológico fundamental consiste en determinar si el trastorno psíquico que observamos corresponde al desarrollo unitario de una personalidad o a la irrupción de un proceso orgánico "que con la interrupción del curso biológico de la vida altera la vida psíquica de modo incurable, irreversiblemente" ${ }^{33} \mathrm{Y}$ es precisamente aquí en donde Jaspers demuestra que este problema tan importante se puede resolver aplicando el método del comprender y el explicar. "Los criterios biográficos del proceso son: la aparición de un elemento nuevo localizado a un breve espacio de tiempo; que éste se acompañe por diversos síntomas conocidos; la ausencia de una causa precipitante o de alguna vivencia suficiente que explique su aparición. Por otra parte, hablamos de desarrollo de una personalidad en cuanto seamos capaces de comprender lo que se ha desarrollado dentro del marco total de la historia vital en todas sus categorías, siempre presuponiendo un sustrato de eventos biológicos normales". ${ }^{44}$ Así pues, si bien en el desarrollo de la personalidad se mantiene la unidad psíquica y por lo tanto es susceptible de ser comprendida, por el contrario, en el proceso la aparición de algo nuevo no comprensible significa una quiebra de sentido en la vida psíquica, que podrá ser susceptible de explicación pero nunca podrá ser comprendida, como erróneamente supuso la escuela psicoanalítica freudiana.

Esta aportación de Jaspers ha sido de la mayor importancia en la historia de la Psiquiatría. Su introducción de la primera parte del método fenomenológico en el estudio de la Psicopatología permitió por vez primera y de manera decididamente científica, distinguir aquellos trastornos que se generan de los acontecimientos y vivencias que conforman la vida psíquica del enfermo (y que por lo tanto son susceptibles de ser comprendidos), de aquellos otros en los que irrumpen elementos psíquicos nuevos -de origen extrapsíquico-; éstos "quiebran" la continuidad de sentido de la vida psíquica sin establecer ninguna conexión con el resto de los elementos psíquicos biográficos y sólo cabe poder explicarlos. Ante estos últimos estamos frente a un fenómeno biológico cuya expresión será psíquica, pero nada más. Donde termina la comprensión, principia la explicación.

A mayor abundamiento, en este último caso, si el hecho no es susceptible de comprensión genética porque no procede de la vida psíquica de la persona, necesariamente su origen es extrapsíquico y, por lo tanto, estamos ante un fenómeno biológico cuya expresión es psíquica, pero nada más. En ausencia de otros métodos para estudiar los trastornos mentales, éste es un instrumento esencial para permitir al psiquiatra distinguir científicamente los trastornos puramente psíquicos de las auténticas enfermedades orgánicas; esto, independientemente de que se conozca o no el sustrato anatomofisiológico sobre el que ellas se asientan.

Para conseguir los objetivos de esta actitud fenomenológica y poder llevar a cabo el análisis de los fenómenos psíquicos: comprenderlos o explicarlos; determinar si se trata de un desarrollo o de un proceso, y establecer sobre bases firmes un diagnóstico, Jaspers considera necesario elaborar una historia patobiográfica lo más completa posible. Esto sólo se obtiene manteniendo un diálogo continuo con el enfermo pues de otra manera no se puede lograr. A lo largo de ese diálogo se podrá conocer paulatinamente la auténtica biografía del enfermo, la trayectoria de su vida, las circunstancias que la rodearon y, sin embargo, sólo hasta cierto punto -puesto que hay muchos límites para la comprensión-, esto permitirá profundizar en las más íntimas vivencias de aquella persona objeto de nuestro estudio. De esta manera se puede conseguir, a veces no sin gran esfuerzo, una descripción aproximada de cómo ha evolucionado su psiquismo. En Psiquiatría no se puede prescindir de la historia patobiográfica del enfermo si en verdad se quiere llegar a un diagnóstico, indispensable para instituir un tratamiento correcto.

Y así lo expresa Jaspers: "Toda vida psíquica es un todo como forma temporal (Zeitgestalt). Captar a un hombre es cosa que exige la contemplación de su vida desde el nacimiento hasta la muerte. Mientras los médicos somáticos como tales sólo tienen que ver con una enfermedad pasajera o crónica... los psiquiatras, en cambio, se han ocupado siempre de toda la vida pasada de sus enfermos con todos sus nexos de naturaleza personal y social. Toda historia clínica correcta conduce a la biografía. La enfermedad psíquica arraiga en el todo de la vida y, para su captación, no se le puede aislar de él". 35 Por lo tanto, en una patobiografía se deben incluir "todos los hechos referentes a un hombre que pueden ser conocidos" ${ }^{35}$ Si bien la historia clínica es esencial en Medicina, en Psiquiatría adquiere particular importancia pues, hasta donde sea posible, deberá aproximarse a reflejar el todo del ser humano: su vida psíquica y su acontecer biológico. Para Jaspers este tema es de capital importancia en Psiquiatría y por ello le dedica un largo capítulo con el que termina la cuarta parte de su Psicopatología General.

Cuando Jaspers escribe su Psicopatología General, la psiquiatría alemana de la época estaba dominada por la figura de Emil Kraepelin, fundador de la Psiquiatría clínica con base biológica empírica, rama de la Medicina y, por lo tanto, incluida entre las ciencias naturales. Por otra parte, en una dimensión distinta y con un enfoque diametralmente opuesto, Sigmund Freud, aplicando al estudio de las neurosis el mé- 
todo comprensivo que pronto extendió al estudio de toda la patología psiquiátrica, proponía su teoría psicoanalítica que muy rápido alcanzó gran difusión y aceptación en ciertos círculos psiquiátricos. Es obvio que Jaspers no podía permanecer ajeno a estas dos corrientes del pensamiento psiquiátrico que no coincidían con el suyo propio y que con modificaciones sustanciales están presentes en la Psiquiatría de hoy.

Emil Kraepelin, en las sucesivas ediciones de su libro, Psiquiatría. Manual para estudiantes y médicos (Psychiatrie. Ein Lehrbruch für Studierende und Ärtze), con gran acierto había propuesto una nueva sistematización de la nosología psiquiátrica que pervive hasta nuestros días. Esta nueva nosografía la elaboró a partir del cuidadoso seguimiento de la evolución de la historia natural de las enfermedades psiquiátricas de infinidad de pacientes, producto de sus propias observaciones y del análisis detallado de cientos de historias clínicas, identificando síntomas, observando sus modificaciones y agrupándolos en síndromes para así llegar a conformar entidades diagnósticas definidas. Con esta sistemática consiguió superar el caos clasificatorio de la Psiquiatría, que prevalecía no sólo en Alemania sino en el mundo entero, al separar un grupo de graves trastornos del pensamiento con características particulares al que denominó "Demencia Precoz", que inevitablemente conducían al deterioro de las funciones psíquicas, es decir a la demencia, de la "Psicosis Maniaco Depresiva"; los trastornos en esta enfermedad eran primariamente propios de la vida afectiva del enfermo, se presentaban en fases que alternaban con periodos de normalidad y su evolución se caracterizaba por no producir deterioro alguno, una vez que desaparecía la fase. No sin cierta resistencia inicial, sobre todo fuera de Alemania, la comunidad psiquiátrica de Europa y finalmente en todo el mundo se aceptó la propuesta de Kraepelin y hoy en día continúa siendo vigente en sus líneas generales y es el pilar que sostiene la nosografía psiquiátrica del siglo XXI.

Jaspers no objeta de manera directa la nosografía de Kraepelin, a la que consideraba que contenía principios claros y muy útiles en cuanto a la clasificación de los trastornos mentales, que superaba con mucho a las que con anterioridad habían propuesto otros autores. Sin embargo, advierte que en ella sólo se pueden incluir aquellas entidades susceptibles de explicación, más no aquellos trastornos que puedan ser comprendidos. Es decir, al panorama diagnóstico de la nosografía de Kraepelin le faltaba la fenomenología.

Por lo tanto, siendo esencial el diagnóstico en la práctica psiquiátrica, Jaspers establece los principios fundamentales que se deben satisfacer para conseguirlo:

"Tiene que ser tal que todo caso pueda ser clasificado solamente en un lugar, que todo caso encuentre un puesto; que la clasificación sea objetivamente obligada, de manera que los diversos investigadores lleguen al mismo ordenamiento de los casos". ${ }^{36}$ Sobre estas bases propone un esquema diagnóstico más amplio que el propuesto por Kraepelin y que divide en tres grupos, a saber: Grupo I. Enfermeda- des somáticas conocidas con perturbaciones mentales (enfermedades cerebrales; afecciones corporales con psicosis sintomáticas; intoxicaciones). Grupo II. Los tres círculos de las grandes psicosis (epilepsia genuina; esquizofrenia; enfermedades maniacodepresivas). Grupo III. Las psicopatías (reacciones anormales independientes de los grupos I y II, las neurosis y los síndromes neuróticos; personalidades anormales y desarrollos). ${ }^{37}$ Esta propuesta nosográfica se parece a la que en 1980 adoptó la Asociación Psiquiátrica Americana en el Diagnostic and Statistical Manual of Mental Disorders (DSM-III) ${ }^{38}$-aunque sólo en sus líneas generales y no en su rigor científico- y que infortunadamente ya se presenta oscurecida y extraviada en el DSM-5.

Pierre Pichot, citando a Kurt Schneider, resume el alcance de la obra de Kraepelin y el pensamiento de Jaspers en cuanto al diagnóstico: "Para Kurt Schneider, el diagnóstico es fundamental: 'Para el pronóstico, el tratamiento y la peritación necesitamos el diagnóstico'. Ahí tenemos la indestructible herencia de Kraepelin: 'Los postes que él plantó siguen de pie. Si (...) se mueven, no es porque se vayan a quebrar, sino porque son elásticos'. Schneider, a su vez, da la definición mil veces citada: 'Diagnosticar es atender al cómo (la forma), y no al qué (el tema, o contenido)... Cuando se mira al contenido, sólo se ve lo biográfico, lo exponible de la existencia. Es lo que sucede en el psicoanálisis y en las nuevas modalidades extremas de la psicopatología existencial. Pero ahí, ciertamente, termina el diagnóstico y por lo tanto la herencia de Kraepelin también. En cambio, la aportación de Jaspers -a quien Kurt Schneider consideraba su único maestro- no da lugar a contradicciones. La psicopatología kraepeliniana, objetivante en exceso, se superó gracias a la 'fenomenología', enunciada con método y programa por Jaspers; objeto de ella eran la vivencia y los modos de vivencia. Perseguía sin embargo, fines diagnósticos, de forma que no entraba en oposición con la psiquiatría clínica". ${ }^{39}$

Por otra parte, las obras de Sigmund Freud alcanzaban gran éxito sobre todo a partir de la publicación, en 1900, de su libro La interpretación de los sueños y su atractiva propuesta de utilizar la comprensión psicológica en el estudio de las neurosis, que ya otros autores habían ensayado antes aunque en otro contexto.

Ya que Freud en su psicoanálisis utilizaba principalmente el método comprensivo como única vía en sus investigaciones y tratamiento, pretendiendo explicar todo el acontecer psíquico sólo con ese método y sin límite alguno con exclusión absoluta del método explicativo, Jaspers, en distintos capítulos de su Psicopatología General, expresa su pensamiento y hace una amplia y detallada crítica de las ideas freudianas.

Como exponerlas todas aquí en detalle sería demasiado extenso, a continuación sólo se citan algunos de los aciertos y objeciones más sobresalientes que Jaspers hace sobre la obra de Freud, en particular aquellas que se refieren a la distinción entre explicación y comprensión. 
Si bien principia Jaspers por reconocer que "Dentro de la psicopatología es un mérito del psicoanálisis la intensificación de la psicología comprensiva", y que "el psicoanálisis ha dirigido con nueva energía la atención a la biografía interna", 40 más adelante señala lo que el psicoanálisis freudiano no respeta.

"Los límites de toda psicología de las relaciones comprensibles son los mismos que deben permanecer necesariamente para el psicoanálisis en la medida en que este último es comprensivo. Esta comprensión cesa primeramente ante la realidad de las características empíricas innatas. Éstas, es verdad, no son nunca definitivamente reconocibles ni pueden ser firmemente establecidas. Pero lo comprensible viene a detenerse ante ellas, como algo impenetrable e inalterable. Los hombres no han nacido iguales sino singulares y comunes en una gradación múltiple y en los aspectos más diversos. En segundo término, la comprensión cesa ante la realidad de las enfermedades orgánicas y de las psicosis, ante la naturaleza elemental de estos hechos. Esta es la realidad decisiva aunque muchas de sus manifestaciones muestren rasgos particulares que al menos en algún aspecto parezcan comprensibles. En tercer lugar, la comprensión cesa ante la realidad de la Existencia misma, de lo que la persona es en sí misma. El esclarecimiento psicoanalítico demuestra aquí ser un pseudo esclarecimiento. Aunque la Existencia misma no se tiene específicamente para la comprensión psicológica, su influencia se deja sentir en los límites que establece para la comprensión psicológica en el mismo punto donde algo que es sólo se manifiesta en el carácter poco concluyente de sentido. El psicoanálisis siempre cierra sus ojos a estas limitaciones y ha querido comprenderlo todo" ${ }^{41}$

En suma, "En Freud se trata realmente de psicología comprensiva y no de la explicación causal como él sostiene... En la confusión de relaciones comprensibles con relaciones causales se basa la inexactitud de la pretensión freudiana de que todo en la vida psíquica, de que todo proceso sea comprensible (determinado con sentido). Sólo se mantiene la exigencia de causalidad ilimitada, no la pretensión de comprensión limitada...". 42

Así expresado el pensamiento de Jaspers sobre Freud, se puede concluir que para él, si bien la taxonomía de Kraepelin contenía principios claros y muy útiles en cuanto a la clasificación de los trastornos mentales, que superaba con mucho a las que con anterioridad habían propuesto otros autores, a las ideas de Freud las calificó desde un principio como poco científicas e inútiles para sustentar sobre ellas las bases de la Psiquiatría.

Jaspers, enemigo de todo dogmatismo, considera que si bien la nosografía es necesaria, indispensable en el quehacer psiquiátrico de todos los días, no puede basarse solamente en la consideración de que "las enfermedades mentales son enfermedades del cerebro", puesto que "esta declaración es tan dogmática como lo sería su negación" ${ }^{43}$ Por lo tanto, ninguna de las dos corrientes, ni la de Kraepelin ni la de
Freud, excluyentes como son, pueden explicar la totalidad del acontecer humano desde una sola perspectiva, como pretenden.

Así pues, es aquí en donde adquiere particular importancia el pensamiento de Jaspers que, por un lado demuestra que la neurobiología, en cuanto ciencia natural que es, sólo puede explicar los fenómenos psíquicos que tengan un origen orgánico, pero nada aporta a la comprensión porque simplemente la excluye. Por otra parte, señala el error de las corrientes psicodinámicas que todo pretenden explicar sin respetar nunca los límites que tiene el método comprensivo. Las dos son dogmas puesto que la afirmación de una excluye totalmente a la otra. Sólo con el comprender y el explicar es posible aproximarse a la totalidad del acontecer humano en la salud y en la enfermedad. Pero, como con un único método no es posible comprenderlo todo, es necesario, antes de emplearlos, reconocer en él sus alcances y limitaciones.

A pesar de que Jaspers, contemporáneo del nacimiento de estas dos corrientes del pensamiento psiquiátrico y que desde entonces hizo en su Psicopatología General la única crítica rigurosamente articulada sobre ellas y que conserva validez plena, no deja de sorprender que haya sido y continúe siendo ignorada por un amplísimo círculo de psiquiatras hasta el día de hoy.

El panorama de la Psiquiatría actual no difiere mucho del que había en la época de Jaspers. Por un lado está el reduccionismo neurobiológico, descendiente directo de Kraepelin, y también la mal llamada psiquiatría neokraepeliniana, cuya máxima expresión han sido los DSM-III, DSM-IV y DSM-5; éstos poco tienen que ver en muchos de sus capítulos con el espíritu riguroso que animó a Emil Kraepelin al construir su propuesta nosográfica. Por otro lado, se encuentra la multitud de teorías analíticas y psicológicas a las que dio origen el psicoanálisis de Freud, con sus múltiples inconsistencias.

Frente a ellas, con nueva actualidad, se alza el pensamiento de Jaspers que con precisión señala las características y los límites que tiene el método explicativo, cuáles son las del método comprensivo y para qué sirve cada uno de ellos. Bien haría la Psiquiatría moderna en atender las enseñanzas de Jaspers para clarificar y profundizar conceptos, establecer límites y avanzar con firmeza en el progreso de esta ciencia.

Los límites que impone un escrito de esta naturaleza impiden hacer una revisión ni siquiera somera de la multitud de conceptos esenciales en la Psiquiatría de hoy, de los que Jaspers se ocupa con todo detalle en su obra. Fueron analizados y clarificados en ella con el método fenomenológico y continúan teniendo validez, tanto desde un punto de vista puramente teórico como en la práctica psiquiátrica de todos los días. Entre ellos destacan la descripción de las características de la percepción y representación normales y patológicas; las pseudopercepciones; las diferencias entre los 
delirios primarios y secundarios; las diferencias entre ilusiones, alucinaciones y pseudoalucinaciones; las características de las vivencias del espacio y del tiempo: su aceleración en la manía y lentificación en la depresión; las diferencias entre ideas delirantes y deliroides; la consciencia de enfermedad y su ausencia; etc,etc.

Ahora bien, si de verdad se quiere progresar en la construcción del saber psiquiátrico, si en verdad se quiere avanzar en el conocimiento del hombre enfermo mental y también del sano, hay que retomar el camino señalado y emprendido por Karl Jaspers. Ciertamente, es necesario continuar el estudio de sus ideas y la profundización de sus conceptos, ir más allá de donde él llegó, teniendo siempre presentes las nuevas aportaciones de las neurociencias. Evitar y combatir toda clase de dogmatismo con sólidos razonamientos tal y como él lo hizo en todo momento a lo largo de su vida, como médico y como filósofo, buscando siempre aproximarnos a la verdad científica. Pero sobre todo aprovechar sus enormes aciertos que, después de cien años, conservan su frescura y vitalidad y nos aproximan mejor que ningún otro método al hombre enfermo mental, objeto primario del interés de la ciencia psiquiátrica.

Siguiendo a Michael Shepherd, es admirable que uno de los pensadores más importantes de su tiempo, antes de dedicarse a la Filosofía, cuando sólo era un joven y brillante médico residente de la Clínica Psiquiátrica de Heidelberg, haya empleado los dos últimos años -de los cuatro que duró su adiestramiento y ejercicio de la Psiquiatría- en escribir una obra tan importante sobre sus fundamentos, los fundamentos de su profesión.

La Psicopatología General de Karl Jaspers ha sido un espléndido regalo a la Psiquiatría Octubre de 2013.

\section{AGRADECIMIENTO}

A Carlos Peniche Ponce por sus valiosas sugerencias para la redacción del texto.

\section{REERENCIAS}

1. Kirkbright S. 'In the clinic'. En: Kirkbright S, Jaspers KA. Biography. Yale, Yale, Yale University Press; 2004; Pág. 62.

2. Ehrlich LH, Ehrlich E, Pepper GB (eds). Origins in scientific research. En: Ehrlich LH, Ehrlich E, Pepper GB, Jaspers K: basic philosophical writings. Selections. Ohio, Ohio: Ohio University Press; 1986; Pág. 5.

3. Jaspers K. Nostalgia y delito. En: Jaspers K. Escritos psicopatológicos. Madrid: Editorial Gredos; 1977; Pág. 9-110.

4. Jaspers K. Delirio celotípico. En: Jaspers K. Escritos psicopatológicos. Madrid: Editorial Gredos; 1977; Pág. 111-181.

5. Jaspers K. Los métodos de medición de la inteligencia y el concepto de demencia. En: Jaspers K. Escritos psicopatológicos. Madrid, Editorial Gredos; 1977; Pág.182-242.

6. Jaspers K. Análisis de las percepciones equívocas. En: Jaspers K. Escritos psicopatológicos. Madrid: Editorial Gredos; 1977; Pág. 243-314.

7. Jaspers K. La corriente de investigación fenomenológica en psicopatología. En: Jaspers K. Escritos psicopatológicos. Madrid: Editorial Gredos; 1977; Pág. 395-412.
8. Jaspers K. Relaciones causales y "comprensibles" entre destino y psicosis en la demencia precoz (esquizofrenia). En: Jaspers K. Escritos psicopatológicos. Madrid: Editorial Gredos; 1977.

9. Jaspers K. Psicopatología general. Buenos Aires: Editorial Beta; 1970.

10. Jaspers K. Psicopatología general. México: Fondo de Cultura Económica; 1993.

11. Emmminhghaus H. Allgemeine Psychopathologie zur einführung in das studium der geistesstörungen ("Psicopatología general como una introducción al estudio de los trastornos mentales"). En: Jaspers K. Introduction. Psicopatología general. Segunda edición en español. México: Fondo de Cultura Económica; 1993; Pág. 48.

12. Störrig $L$. Vorlesungen über psychopathologie in ihrer bedeutung für die normale psychologie ("Conferencias sobre la psicopatología y su importancia para la psicología normal"). En: Jaspers K. Introducción. Psicopatología general. Segunda edición en español. México: Fondo de Cultura Económica; 1993; Pág. 48.

13. Jaspers K. Introduction. En: General psychopathology. 2 vols Trans. Hoenig J, Hamilton MW. Baltimore: Johns Hopkins University Press; 199; Pág. 39.

14. Jaspers K. Introduction. En: General psychopathology. 2 vols Trans. Hoenig J, Hamilton MW. Baltimore: Johns Hopkins University Press; 1997; Pág. 40.

15. Jaspers K. Introducción. En: Jaspers K. Psicopatología general. México: Fondo de Cultura Económica; 1993; Pág. 48.

16. Jaspers K. Introduction. En: General psychopathology. 2 vols Trans. Hoenig J, Hamilton MW. Baltimore: Johns Hopkins University Press; 1997; Pág. 2.

17. Shepherd M. Review of general psychopathology, by Karl Jaspers. Brit J Psychiatry 1982;141:310-312.

18. Ghaemi SN. The concepts of psychiatry. Baltimore: Johns Hopkins University Press; 2003.

19. Shepherd M. Review of general psychopathology, by Karl Jaspers. Brit J Psychiatry 1982;141:310-312.

20. Jaspers K. Introducción. En: Jaspers K. Psicopatología general. México: Fondo de Cultura Económica; 1993; Pág. 55-56.

21. Doerr-Zegers O, Pelegrina-Cetrán. Karl Jaspers' General psychopthology in the framework of clinical practice. En: Stanghellini G, Fuchs Th. One century of Karl Jaspers' General psychopathology. Oxford University Press; 2013; Pág. 58.

22. Jaspers K. Introducción. En: Jaspers K. Psicopatología General. México: Fondo de Cultura Económica; 1993. Pág. 58.

23. Jaspers K. Subjective phenomena of morbid psychic life. En: General psychopathology. 2 vols Trans. Hoenig J, Hamilton MW. Baltimore: Johns Hopkins University Press; 199: Pág. 55.

24. Jaspers K. Introduction. En: General psychopathology. 2 vols Trans. Hoenig J, Hamilton MW. Baltimore: Johns Hopkins University Press; 1997; Pág. 17.

25. Jaspers K. Introducción. En: Jaspers K. Psicopatología general. México: Fondo de Cultura Económica; 1993; Pág. 35.

26. Dilthey W. Ideas acerca de una psicología descriptiva y analítica. En: Obras de Wilhelm Dilthey. Tomo VI. México: Fondo de Cultura Económica; 1978.

27. Jaspers K. Introducción. En: Jaspers K. Psicopatología general. México: Fondo de Cultura Económica; 1993; Pág.36.

28. Jaspers K. Las relaciones comprensibles de la vida psíquica (psicología comprensiva). En: Jaspers K. Psicopatología general. México: Fondo de Cultura Económica; 1993; Págs. 342-343.

29. Jaspers K. Meaningful psychic connections. En: General psychopathology. 2 vols Trans. Hoenig J, Hamilton MW. Baltimore: Johns Hopkins University Press; 1997; Pág. 304.

30. Jaspers K. Las relaciones comprensibles de la vida psíquica (psicología comprensiva). En: Jaspers K. Psicopatología general. México: Fondo de Cultura Económica; 1993; Pág. 344.

31. Jaspers K. Meaningful psychic connections. En: General psychopathology. 2 vols Trans. Hoenig J, Hamilton MW. Baltimore: Johns Hopkins University Press; 1997; Pág. 304. 
32. Jaspers K. Las relaciones comprensibles de la vida psíquica (psicología comprensiva). En: Jaspers K. Psicopatología general. México: Fondo de Cultura Económica; 1993; Pág. 345.

33. Jaspers K. El curso de la vida (Biografía). En: Jaspers K. Psicopatología general. México: Fondo de Cultura Económica; 1993; Pág. 776.

34. Jaspers K. Biographical study (Biographik). En: General psychopathology. 2 vols Trans. Hoenig J, Hamilton MW. Baltimore: Johns Hopkins University Press; 1997; Pág. 702.

35. Jaspers K. El curso de la vida (biografía). En: Jaspers K. Psicopatología general. México: Fondo de Cultura Económica; 1993; Págs. 743 y 776.

36. Jaspers K. La síntesis de los cuadros nosológicos. En: Jaspers K. Psicopatología general. México: Fondo de Cultura Económica; 1993; Pág. 672.

37. Jaspers K. La síntesis de los cuadros nosológicos. En: Jaspers K. Psicopatología general. México: Fondo de Cultura Económica; 1993; Pág. 673.
38. Ghaemi SN. The concepts of psychiatry. Baltimore: The Johns Hopkins University Press: 2007; Pág 70.

39. Pichot P. Un siglo de psiquiatría. París: Editions Roger Dacosta; 1983; Pág 100.

40. Jaspers K. Relaciones comprensibles. En: Jaspers K. Psicopatología general. México: Fondo de Cultura Económica; 1993; Pág. 404.

41. Jaspers K. Meaningful connections. En: General psychopathology. 2 vols Trans. J. Hoenig and M.W. Hamilton. Baltimore: Johns Hopkins University Press; 1997. Pág. 363.

42. Jaspers K. Sobre el sentido y el valor de las teorías. En: Jaspers K. Psicopatología general. México: Fondo de Cultura Económica; 1993; Pág. 601.

43. Jaspers K. The causal connections of psychic life. En: General psychopathology. 2 vols Trans. Hoenig J, Hamilton MW. Baltimore: Johns Hopkins University Press; 1997; Pág. 459. 